\title{
Tumores craneales radioinducidos: serie clínica y revisión de la literatura
}

\author{
J. M. Garbizu; O. Mateo-Sierra; J. M. Pérez-Calvo; B. Iza y F. Ruiz-Juretschke
}

Servicio de Neurocirugía. Hospital General Universitario Gregorio Marañón. Madrid.

Resumen

La inducción de neoplasias constituye una complicación bien conocida, aunque poco frecuente, de la irradiación craneal. Los meningiomas son las neoplasias craneales radioinducidas (RI) más frecuentes, seguidas de los gliomas y los sarcomas, siendo extremadamente raros otros tipos tumorales tales como los hemangioblastomas.

Presentamos 7 pacientes con tumores craneales secundarios a radioterapia, diagnosticados en nuestro hospital entre los años 1990 y 2006 . Se realizó una revisión retrospectiva de sus datos clínicos. Todos los pacientes habían sido irradiados en la infancia como tratamiento de otra enfermedad, y cumplían los criterios de neoplasia $R I$.

Cuatro pacientes desarrollaron meningiomas, mientras que los 3 restantes presentaron otros tumores (un glioblastomas multiforme, un hemangioblastoma y un osteosarcoma de partes blandas). En los siete casos se logró una resección quirúrgica completa. El diagnóstico preoperatorio basado en resonancia magnética (RM) coincidió con el diagnóstico histológico en seis casos. Los factores de riesgo más importantes para desarrollar una neoplasia RI son, según la literatura, la edad en el momento de la irradiación y la dosis administrada. El diagnóstico diferencial de estas neoplasias se plantea con aquellas lesiones que aparezcan tras la irradiación craneal, especialmente las recidivas, ya que el desarrollo de neoplasias RI constituye una complicación muy poco frecuente. El pronóstico de esta enfermedad, incluso tras resecciones completas, depende del diagnóstico histológico del tumor RI.

PALABRAS CLAVE: Tumor radioinducido. Meningioma. Hemangioblastoma radioinducido.

Radiation-induced cranial tumors: clinical series and literature review

Recibido: 4-01-08. Aceptado: 13-03-08
Radiation induced tumors are well-known but rare complications of radiotherapy. Meningiomas are the most common radiation-induced (RI) cranial tumors, followed by gliomas and sarcomas, while other tumors as haemangioblastomas remain extremely exceptional.

We present 7 patients with RI brain tumors diagnosed and treated at our institution between 1990 and 2006. Retrospective review of their clinical charts is supplied. All patients were irradiated during childhood as a treatment for another disease, and fulfilled the criteria of RI neoplasia.

Four patients developed meningiomas and three developed other tumors (one glioblastoma, one softtissue sarcoma and one hemangioblastoma). In all cases a complete surgical removal was achieved. Preoperative assessment based on MRI supplied the correct diagnosis in six patients.

The most important risks factors described in the literature for developing RI tumors are the age at which radiotherapy was administered and the dose of radiation applied. Differential diagnosis of RI tumors includes any tumor appearing after radiotheraphy, especially recurrences of the primary disease, as RI neoplasias are a rare complication. Even in cases with complete surgical resection, prognosis of this clinical entity is basically related to the histology of the RI tumor.

KEY WORDS: Radiation-induced tumor. Meningiomas. Radiation-induced hemangioblastoma.

Introducción

La irradiación craneal constituye una herramienta terapéutica valiosa para el tratamiento de determinadas lesiones cerebrales. Sin embargo, puede presentar múltiples efectos colaterales. Estos efectos indeseables pueden tener lugar a corto plazo (náuseas, vómitos, tinnitus,

Abreviaturas. $R I$ : radioinducida. RM: resonancia magnética. $R T$ : radioterapia. 

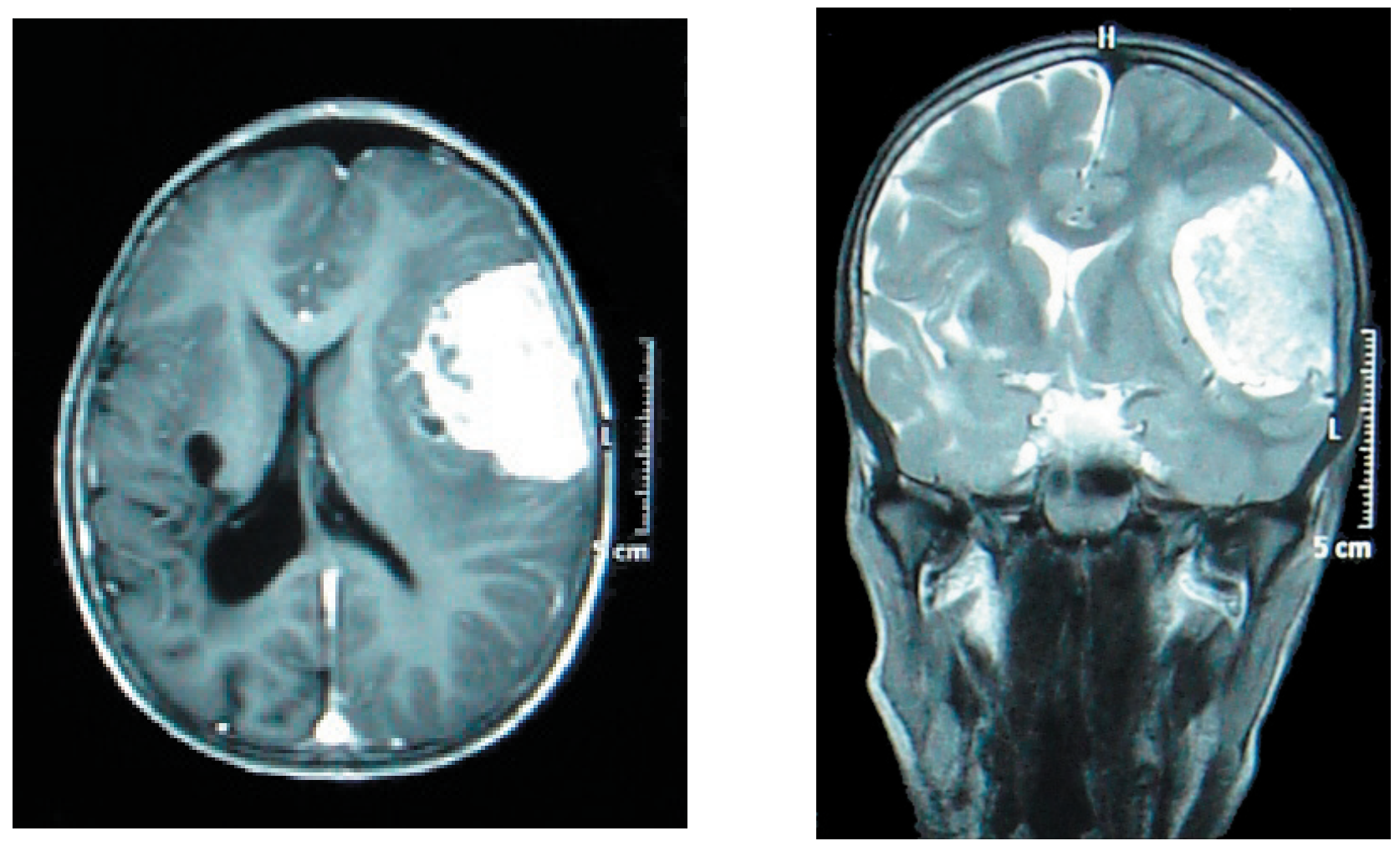

Figuras 1 y 2. RM en cortes axiales y coronales de meningioma frontotemporal radioinducido (caso 5).

alteraciones cutáneas, alopecia) o a largo plazo (disminución de la agudeza visual, síndrome orgánico cerebral, enfermedad vascular oclusiva, panhipopituitarismo, neoplasias) ${ }^{1}$. La posibilidad de inducción de neoplasias craneales constituye un efecto secundario poco frecuente, pero bien documentado.

El diagnóstico de neoplasia RI debe realizarse en base a una serie de criterios diagnósticos previamente estableci$\operatorname{dos}^{5,6,16}$ :

1. Localización en el campo de irradiación del tratamiento previo.

2. No estar presente en el momento de la irradiación.

3. Intervalo de tiempo suficiente entre la irradiación y la aparición de la neoplasia secundaria (generalmente, varios años).

\section{Histología diferente.}

5. Ausencia de enfermedades o condiciones genéticas predisponentes (síndrome de Li Fraumeni, neurofibromatosis, xeroderma pigmentosum, inmunodeficiencia).

Los tumores craneales RI más frecuentes son los meningiomas, siendo menos frecuentes otros tipos histológicos, como los gliomas y los sarcomas.

Presentamos una serie de pacientes con tumores que cumplían los criterios de neoplasia RI y realizamos una revisión de la literatura. Se describe el caso excepcional de un paciente con un hemangioblastoma RI.

\section{Pacientes y métodos}

Presentamos 7 casos de pacientes diagnosticados de neoplasias craneales secundarias a radioterapia (RT) entre los años 1990 y 2006. Todos los casos cumplían los criterios de neoplasia RI previamente expuestos. Se realizó una revisión retrospectiva de sus historias clínicas, pruebas de imagen y estudios anatomopatológicos. Para ello, se recogieron datos relacionados con la edad, tanto en el momento de la irradiación como en el momento del diagnóstico del tumor secundario, sexo, enfermedad primaria que motivó la radioterapia, dosis de radiación recibida, tiempo transcurrido entre el momento de la irradiación y el diagnóstico del tumor secundario (periodo de inducción), tipo histológico y localización de la lesión.

\section{Resultados}

Todos los pacientes (Tabla 1) recibieron dosis variables de radiación durante la infancia. De entre ellos, 2 recibieron dosis bajas de radiación ( $<10 \mathrm{~Gy}$ ), en el contexto de la terapia de Adamson-Kiëmbock para el tratamiento de la tinea capitis (casos 1 y 2). El resto fueron sometidos a dosis altas de radiación $(>20 \mathrm{~Gy})$ para erradicar diferentes tipos de lesiones tumorales: un glioma de nervio óptico (caso 3), un meduloblastoma (caso 4), un astrocitoma profundo grado II (caso 5), un germinoma de III ventrículo (caso 6) y un 
Tabla 1

Resumen de los principales datos clínicos de los pacientes de la serie presentada

\begin{tabular}{|c|c|c|c|c|c|c|c|c|}
\hline & & \multicolumn{3}{|c|}{ Enfermedad Primaria } & \multicolumn{4}{|c|}{$\underline{\text { Neoplasia Secundaria }}$} \\
\hline Caso & $\begin{array}{l}\text { Edad/ } \\
\text { Sexo }\end{array}$ & Diagnóstico & $\begin{array}{l}\text { Dosis de } \\
\text { radiación } \\
(G y)\end{array}$ & $\begin{array}{l}\text { Edad } \\
\text { (años) }\end{array}$ & $\begin{array}{l}\text { Periodo } \\
\text { inducción } \\
\text { (años) }\end{array}$ & Diagnóstico AP & Multiplicidad & Localización \\
\hline 1 & $50 / \mathrm{M}$ & Tinea capitis & $<10$ & 5 & 45 & $\begin{array}{c}\text { Meningioma } \\
\text { transicional } \\
\text { (atípico) }\end{array}$ & $\mathrm{Si}$ & $\begin{array}{c}\text { Surco } \\
\text { olfatorio, hoz } \\
\text { y convexidad } \\
\text { occipital }\end{array}$ \\
\hline 2 & $47 / \mathrm{V}$ & Tinea capitis & $<10$ & 4 & 43 & $\begin{array}{l}\text { Meningioma } \\
\text { meningiotelial }\end{array}$ & No & $\begin{array}{l}1 / 3 \text { anterior de } \\
\text { la hoz }\end{array}$ \\
\hline 3 & $22 / \mathrm{M}$ & $\begin{array}{c}\text { Glioma de nervio } \\
\text { óptico }\end{array}$ & 50 & 6 & 16 & $\begin{array}{l}\text { Glioblastoma } \\
\text { multiforme }\end{array}$ & No & $\begin{array}{l}\text { Lóbulo } \\
\text { temporal } \\
\text { izquierdo }\end{array}$ \\
\hline 4 & $36 / \mathrm{V}$ & Meduloblastoma & 60 & 14 & 22 & Hemangioblastoma & No & Tentorio \\
\hline 5 & $16 / \mathrm{V}$ & $\begin{array}{l}\text { Astrocitoma } \\
\text { hemisférico } \\
\text { (grado II) }\end{array}$ & 50 & 1 & 15 & $\begin{array}{l}\text { Meningioma } \\
\text { meningotelial }\end{array}$ & No & $\begin{array}{l}\text { Convexidad } \\
\text { frontotemporal } \\
\text { izquierda }\end{array}$ \\
\hline 6 & $22 / \mathrm{M}$ & $\begin{array}{l}\text { Germinoma de } \\
\text { III ventrículo }\end{array}$ & 45 & 10 & 12 & $\begin{array}{c}\text { Meningioma } \\
\text { transicional } \\
\text { (atípico) }\end{array}$ & No & Hoz cerebral \\
\hline 7 & $13 / \mathrm{V}$ & $\begin{array}{c}\text { Astrocitoma } \\
\text { pilocítico } \\
\text { de cerebelo }\end{array}$ & 40 & 8 & 5 & $\begin{array}{c}\text { Osteosarcoma de } \\
\text { partes blandas }\end{array}$ & No & $\begin{array}{l}\text { Región } \\
\text { retromastoidea } \\
\text { derecha }\end{array}$ \\
\hline
\end{tabular}

astrocitoma pilocítico de cerebelo (caso 7).

En el momento de la irradiación todos los pacientes presentaban edades comprendidas entre 1 y 14 años (media: 7,5 años). Todos ellos recibieron RT como parte del tratamiento de su enfermedad primaria. De entre aquellos casos que presentaban una enfermedad primaria neoplásica, los casos 3, 4, 6 y 7 fueron sometidos a cirugía y RT, mientras que el caso 5 (Figuras 1 y 2) sólo recibió RT. Éstos se encontraban en situación de remisión completa en el momento del diagnóstico de su neoplasia secundaria.

Por lo que se refiere al periodo de inducción, aquéllos con el diagnóstico de meningioma presentaron valores comprendidos entre 12 y 45 años (media: 30 años); para el caso 4, con diagnóstico anatomopatológico de hemangioblastoma, el periodo de inducción fue de 22 años; y, finalmente, para los casos 3 y 7 , el periodo de inducción fue de 16 y 5 años, respectivamente.

Sólo un paciente (caso 1) presentaba numerosas lesiones en el momento del diagnóstico. Se trataba de una mujer con múltiples meningiomas (a nivel de la hoz cerebral, el surco olfatorio y la convexidad occipital) que había sido sometida a dosis bajas de radiación durante la infancia para tratamiento de una tinea capitis.

El diagnóstico preoperatorio por RM se correspondía con el diagnóstico anatomopatológico tras la resección tumoral, salvo para el caso 4. Este paciente había sido tratado durante la infancia por un meduloblastoma mediante cirugía y RT. En el curso de una RM craneal de control fue diagnosticado de lesión compatible con meningioma de tentorio (Figura 3). Sin embargo, el diagnóstico anatomopatológico definitivo mediante técnicas inmunohistoquímicas demostró que realmente se trataba de 


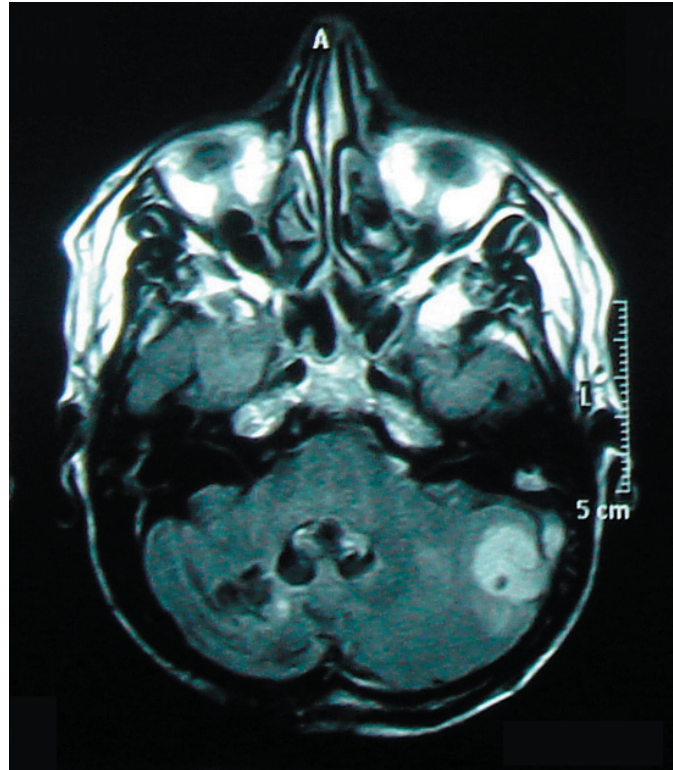

Figura 3. RM de hemangioblastoma cerebeloso (caso 4). Diagnóstico inicial de meningioma de tentorio.

un hemangioblastoma (Figuras 4 y 5).

Todos los pacientes fueron sometidos a cirugía, pudiéndose realizar resecciones tumorales completas en todos los casos. El caso 3, además recibió quimioterapia con temozolamida.

El periodo de seguimiento fue superior a los 24 meses, excepto para los casos 4 y 5 . No se objetivaron recidivas, salvo para los casos 1 y 3 . La paciente con el glioblastoma multiforme (caso 3) falleció 6 meses después del diagnóstico de la neoplasia secundaria.

Aquellos casos que recibieron dosis bajas de radiación durante la infancia (casos 1 y 2) presentaban un periodo de inducción más largo con respecto a aquéllos que habían recibido dosis más altas (valores medios, 44 años vs 17,7 años).

\section{Discusión}

La aparición de neoplasias tras el tratamiento con radioterapia es una complicación rara, que aparece con una frecuencia que oscila entre el $0,03 \%$ y el $0,3 \%$ de los casos tratados $^{12}$. El sistema nervioso central es sensible al desarrollo de este tipo de neoplasias, especialmente cuando ésta se administra en la infancia ${ }^{7,13,15}$. Presentamos 7 casos de pacientes que recibieron radioterapia en edades precoces, 2 de ellos en el contexto de la terapia de Adamson-Kienböck para tratamiento de la tinea capitis y el resto para tratar diferentes tipos de lesiones tumorales.

En cuanto al mecanismo de producción, se postula que puede relacionarse con el daño inducido por las radiaciones ionizantes en el DNA a nivel de oncogenes y genes

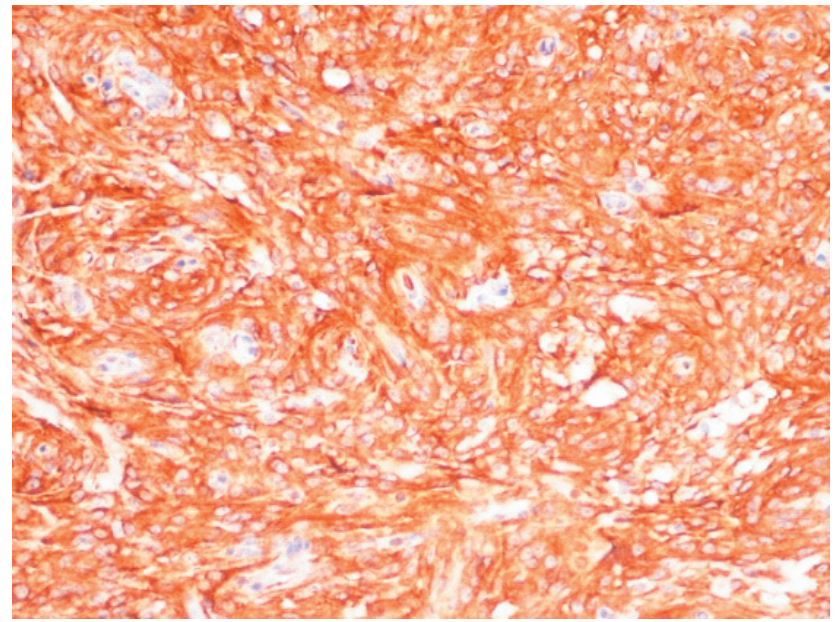

Figura 4. Inmunohistoquímica positiva para Vimentina del hemangioblastoma radioinducido (caso 4).

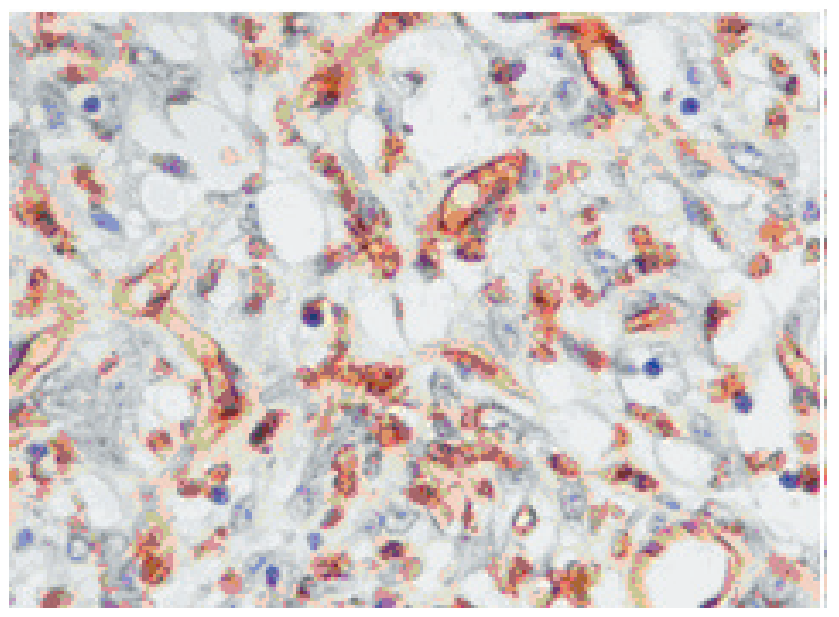

Figura 5. Corte histológico positivo para factor VIII de hemangioblastoma radioinducido (caso 4).

oncosupresores. Por lo que se refiere a los gliomas y los sarcomas RI, se comprueba de forma casi constante la mutación en el gen p533,16. En cuanto a los meningiomas, se objetiva la presencia de múltiples reordenamientos cromosómicos, siendo menos frecuentes con respecto a los casos no RI la inactivación del gen NF2 y la pérdida del cromosoma 22, y más frecuentes otras aberraciones cromosómicas, incluyendo la pérdida de todo o parte del cromosoma $1 \mathrm{p}^{1}$. Esta anomalía típicamente se asocia a meningiomas no RI que presentan un comportamiento más agresivo ${ }^{1}$.

Será fundamental realizar un diagnóstico diferencial con todas aquellas lesiones cerebrales que tienen lugar tras la administración de RT en el contexto de una enfermedad neoplásica ${ }^{3,10,11,14}$. Ante el desarrollo de una nueva lesión ocupante de espacio habrá que considerar como primera posibilidad la recidiva tumoral, seguida de la radionecrosis 
y, más raramente, la aparición de un tumor secundario. Sin embargo, las características clínicas y radiológicas por TC y RM de estas entidades son semejantes, lo cual dificulta considerablemente el diagnóstico diferencial. Las técnicas de PET y SPECT han sido ampliamente utilizadas para valorar la actividad metabólica de las lesiones neoplásicas, así como del tejido cerebral en diferentes situaciones patológicas. La hipercaptación de radiotrazadores se asocia a la presencia de neoplasia, mientras que la ausencia de acumulación de estas sustancias indica la existencia de una radionecrosis cerebral ${ }^{11,14}$. Sin embargo, se considera que estas técnicas no siempre permiten diferenciar ambos tipos de lesiones, y, por lo tanto, el diagnóstico definitivo depende del análisis anatomopatológico del tejido en cuestión.

Describimos 7 pacientes con 4 estirpes histológicas diferentes: 4 meningiomas (casos 1, 2, 5 y 6); 1 glioblastoma multiforme (caso 3); 1 hemangioblastoma (caso 4) y 1 osteosarcoma de partes blandas (caso 7).

Los tipos histológicos tumorales tradicionalmente descritos en relación con la administración de RT son los meningiomas, seguidos de los gliomas y, por último, de los sarcomas ${ }^{4,6,7,15,17}$. Describimos el caso de un paciente con el diagnóstico de hemangioblastoma que cumplía los criterios de neoplasia RI y resaltamos la rareza de este hallazgo dado que sólo hemos encontrado un caso semejante descrito en la literatura9.

Se describen diferentes factores asociados a un mayor riesgo de desarrollar neoplasias secundarias a RT: tipo y dosis de radiación, vulnerabilidad tisular, enfermedad subyacente y régimen quimioterápico asociado ${ }^{2,4,5,17,18}$.

Los factores predisponentes considerados como más importantes son la edad en el momento de la irradiación y la dosis de radiación recibida ${ }^{1,11}$. En nuestra serie todos los pacientes habían sido irradiados durante la infancia. Harrison y cols. ${ }^{8}$ clasificaron los meningiomas RI en 3 grupos basándose en la dosis de radiación administrada: dosis baja $(<10 \mathrm{~Gy})$, dosis moderada (10-20 Gy) y dosis alta $(>20$ Gy).

Según la literatura, el riesgo relativo de desarrollar un tumor secundario cuando se administra RT durante la infancia oscila entre 2,6 y $38,8^{7,13,15}$, mientras que en el adulto este riesgo se estima entre 9,38 y $16^{4,17}$. Por otro lado, se considera que el periodo de inducción es inversamente proporcional a la dosis de radiación recibida ${ }^{1,8}$. En nuestra casuística aquellos pacientes que recibieron dosis bajas de radiación $(<10 \mathrm{~Gy})$ presentaban periodos de inducción más largos que aquellos que recibieron dosis altas (valores medio, 44 vs. 17,7 años).

Se ha postulado que los meningiomas tienen lugar con mayor frecuencia tras recibir dosis bajas de radiación, mientras que los gliomas y los sarcomas ocurren con mayor frecuencia en relación con dosis de radiación altas.
Según Al-Mefty y cols. ${ }^{1}$ esto es una conclusión errónea ya que, antiguamente, los meningiomas RI se diagnosticaban en individuos que habían sido irradiados con dosis bajas en relación con el método de Adamson-Kienböck. Teniendo en cuenta que la radiación administrada con esta técnica no penetraba en profundidad más allá de las meninges, el riesgo de desarrollar gliomas o sarcomas intracraneales era muy inferior. Actualmente, la mayoría de los casos de meningiomas RI son diagnosticados en pacientes sometidos a RT con altas dosis para tratamiento de enfermedades neoplásicas, al igual que en aquéllos que presentan gliomas o sarcomas secundarios.

En cuanto a los meningiomas secundarios a RT, éstos presentan una serie de características diferenciales: mayor frecuencia de comportamiento agresivo, de histología atípica, mayor incidencia de lesiones múltiples y mayor frecuencia de recidivas tras cirugía ${ }^{1}$.

La tasa de recidiva para los meningiomas RI es superior a la de los casos espontáneos según la literatura ${ }^{8}$. Para determinados autores esto puede ser atribuido a la alta frecuencia de invasión del hueso que dificulta la extirpación completa de estas lesiones ${ }^{8}$. Por lo que se refiere a la multiplicidad de estas lesiones, entre un 0,6 y un $2,5 \%$ de los meningiomas no RI presentan múltiples localizaciones, mientras que si nos referimos a los meningiomas RI la frecuencia asciende hasta un $29 \% 1$.

Los sarcomas RI presentan un comportamiento más agresivo $\mathrm{y}$, por tanto, peor pronóstico que aquéllos con semejantes características histológicas que se desarrollan sin RT previa ${ }^{11}$. Describimos el caso de un paciente con un osteosarcoma RI de partes blandas localizado en región retromastoidea derecha que presentó una evolución favorable tras la resección completa de la lesión.

$\mathrm{Al}$ igual que para los tumores primarios, el tratamiento fundamental consiste en una extirpación quirúrgica lo más completa posible. Valorando las otras modalidades de tratamiento complementarias, cabe resaltar que la RT se encuentra limitada por la dosis de radiación previamente administrada. Sin embargo, las nuevas técnicas que permiten calcular de forma precisa la dosis de radiación distribuida permiten salvar en parte esta limitación, además de contribuir con gran probabilidad a reducir la incidencia de estos tumores secundarios a RT.

\section{Conclusiones}

Las neoplasias craneales secundarias constituyen una complicación poco frecuente tras la administración de RT. Será necesario realizar un diagnóstico diferencial con otras lesiones ocupantes de espacio más frecuentes tales como la recidiva tumoral o la radionecrosis.

Los factores predisponentes más importantes relacionados con el desarrollo de estas lesiones son la edad en el 
momento de la irradiación y la dosis de radiación administrada. Cuanto más joven es el paciente y cuanto mayor es la dosis de radiación administrada, mayor será el riesgo de desarrollar tumores secundarios.

La sofisticación de las técnicas de RT que se emplean en la actualidad permitirá con toda probabilidad reducir la incidencia de este tipo de lesiones.

El hemangioblastoma de fosa posterior debe incluirse entre los posibles diagnósticos de neoplasia RI.

\section{Bibliografía}

1. Al-Mefty, O., Topsakal, C., Pravdenkova, S., Sawyer, J., Harrison, M.J.: Radiation-induced meninigiomas: clinical, pathological, cytokinetic, and cytogenetic characteristics. J Neurosurg. 2004; 100: 1002-1013.

2. Bliss, P., Kerr, GR., Gregor, A.: Incidence of second brain tumours after pituitary irradiation in Edinburgh 19621990. Clin Oncol. 2004; 6: 361-363.

3. Brachman, D.G., Hallahan, D.E., Beckett, M.A., Yandell, D.W., Weichselbaum, R.R.: p53 gene mutations and abnormal retinoblastoma protein in radiation-induced human sarcomas. Cancer Res. 1991; 51: 6393-6396.

4. Brada, M., Ford, D., Ashley, S., Bliss, J.M., Crowley, S., Mason, M., Rajan, B., Traish, D.: Risk of second tumour after conservative surgery and radiotherapy for pituitary adenoma. BMJ. 1992; 304: 1343-1346.

5. Cahan, W.G., Woodard, H.Q., Higinbotham, N.L., Stewart, F.W., Coley, B.L.: Sarcoma arising in irradiated bone - report of 11 cases. Cancer 1948; 1: 3-29.

6. Chang, S.M., Barker, F.G., Larson, D.A., Bollen, A.W., Prados, M.D.: Sarcomas subsequent to cranial irradiation. Neurosurgery. 1995; 36: 685-690.

7. Dodick, D.W., Mokri, B., Shaw, E.G., Miller, G.M., Unni, K.K.: Sarcomas of calvarial bones: Rare remote effect of radiation therapy for brain tumors. Neurology. 1994; 44: 908-912.

8. Harrison, M.J., Wolfe, D.E., Lau, T.S.: Radiation-induced meningiomas: experience at the Mount Sinai Hospital and review of the literature. J Neurosurg. 1991; 75: 564-574.

9. Legerlotz, C.: Radiation-induced malignant hemangioblastoma. Zentr. Allgem. Pathol. Pathol. Anat. 2001; 104: 59-66.
10. Nishio, S., Morioka, T., Fukui, M.: Radiation injury of the brain. Crit Rev Neurosurg. 1997; 7: 408-415.

11. Nishio, S., Morioka, T., Inamura, T., Takeshita, I., Fukui, M., Sasaki, M., Nakamura, K., Wakisaka, S.: Radiation-induced brain tumours: potential late complications of radiation therapy for brain tumours. Acta Neurochir (Wien). 1998; 140: 763-770.

12. Patel, S.R.: Radiation-induced sarcoma. Curt. Treat. Options Oncol. 2000; 1: 258-261.

13. Ron, E., Modan, B., Boice, J.D., Alfandary, E., Stovall, M., Chetrit, A., Katz, L.: Tumors of the brain and nervous system after radiotherapy in childhood. N Engl J Med. 1988; 319: 1033-1039.

14. Sasaki, M., Ichiya, Y., Kuwabara, Y., Yoshida ,T., Inoue, T., Morioka, T., Hisada, K., Fukui, M., Masuda, K.: Hyperperfusion and hypermetabolism in brain radiation necrosis with epileptic activity. J Nucl Med 1996; 37: 11741176.

15. Soffer, D., Pittaluga, S., Feiner, M., Beller, A. J.: Intracranial meningiomas following low-dose irradiation to the head. J Neurosurg. 1983; 59: 1048-1053.

16. Tada, M., Sawamura, Y., Abe, H., Iggo, R.: Homozygous p53 gene mutation in a radiation-induced glioblastoma 10 years after treatment for an intracranial germ cell tumor: case report. Neueosurgery. 1997; 40: 393-396.

17. Tsang, R.W., Laperriere, N.J., Simpson, W.J., Brierley, J., Panzarella, T., Smyths, H.S.: Glioma arising after radiation therapy for pituitary adenoma. A report of four patients and estimation of risk. Cancer. 1993; 72: 2227-2233.

18. Tucker, M.A., D’Angio, G.J., Boice, J.D., Strong, L.C., Li, F.P., Stovall, M., Stone, B.J., Breen, D.M., Lombardi, F., Newton, W., Hoover, R.N., Fraumeni, J.F.: Bone sarcomas linked to radiotherapy and chemotherapy in children. N Engl J Med. 1987; 317: 588-593.

Garbizu, J.M.; Mateo-Sierra, O.; Pérez-Calvo, J.M.; Iza, B.; Ruiz-Juretschke, F.: Tumores craneales radioinducidos: serie clínica y revisión de la literatura. Neurocirugía 2008; 19: 332-337.

Correspondencia postal: José Manuel Garbizu Vidorreta. Servicio de Neurocirugía. Hospital General Universitario "Gregorio Marañón”. C/Dr. Esquerdo 46. 28007-Madrid 